\title{
HIV-2 infection is associated with preserved GALT homeostasis and epithelial integrity despite ongoing mucosal viral replication
}

\author{
SM Fernandes ${ }^{1,2}$, AR Pires ${ }^{1}$, P Matoso $^{1,6}$, C Ferreira $^{2}$, H Nunes-Cabaço $^{1}$, L Correia ${ }^{2}$, E Valadas ${ }^{2,3}$, J Poças $^{4}$,

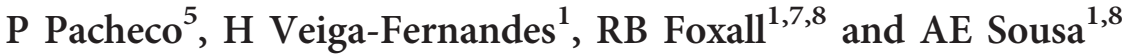

The mechanisms that enable preservation of gut mucosal integrity during persistent viral replication and inherent inflammation remain unclear. Here, we investigated, for the first time, gut homeostasis in HIV-2 infection, a naturally occurring form of attenuated HIV disease. We found viral replication in both sigmoid and ileum of asymptomatic HIV-2 + patients (range: 240-851 circulating CD4 + T-cells per $\mu$ l) despite their undetectable viremia, accompanied by interferon$\gamma$-producing CD8 T-cell expansion, irrespective of antiretroviral treatment. Nevertheless, there was no CD4 T-cell depletion, and Foxp3 + and IL-17- or IL-22-producing CD4 T-cell numbers were unaffected. Moreover, IL-22-producing innate lymphoid cells and IL-22-induced antimicrobial peptides and mucins were maintained. In agreement, the epithelium histology was preserved, including tight junction protein zonula occludens (ZO-1) levels. Furthermore, in vitro infection of colon epithelia with primary isolates revealed no HIV-2 impact on ZO-1 expression. Notably, sigmoid transcriptional levels of CCL20 and CCL28 were significantly increased, in direct correlation with GM-CSF, indicating a local response able to enhance CD4 T-cell recruitment. In conclusion, maintenance of mucosal integrity in HIV-2 infection was associated with T-cell recruitment responses, potentially counteracting CD4 T-cell depletion due to HIV-2 replication. These data have unique implications for the design of therapies targeting gut homeostasis in HIV-1 infection and other chronic inflammatory settings.

\section{INTRODUCTION}

The gut epithelium represents the largest barrier in the body, and its continuous cross-talk with gut microbiota is critical for health. ${ }^{1}$ Mucosal homeostasis depends on the interplay between epithelia and the immune system. ${ }^{2}$ These finely balanced pro-inflammatory and tolerogenic mechanisms are vital for preventing pathogenic infections and fine tuning the microbiome. ${ }^{3}$ T- and $\mathrm{B}$ cells are viewed as the main immunological players, although innate lymphoid cells (ILCs) have been recently shown to have a prominent role. ${ }^{4}$ The cytokine interleukin-22 (IL)-22, produced by type 3 ILCs (ILC3) and/or T-cells, is crucial to this process, regulating epithelial proliferation and tissue regeneration, as well as the production of antimicrobial peptides. ${ }^{5}$ Equally important for protection against infections is IL-17, a pro-inflammatory cytokine that can also be produced by ILC 3 and CD4 T-cells. Of note, maintenance of gut homeostasis requires a balance between IL-17 production and suppressive mechanisms mainly driven by regulatory T-cells (Tregs). ${ }^{6,7}$

Disruption of mucosal integrity is considered a main determinant of gut and systemic diseases, such as HIV/AIDS. ${ }^{8}$ HIV-1 and Simian Immunodeficiency Virus (SIV) have been shown to impact on the bowel mucosa in acute infection, leading to a depletion of CD4 T-cells from the lamina propria

${ }^{1}$ Instituto de Medicina Molecular, Faculdade de Medicina, Universidade de Lisboa, Lisbon, Portugal. ${ }^{2}$ Hospital de Santa Maria, Centro Hospitalar Lisboa Norte-EPE, Lisboa, Portugal. ${ }^{3}$ Clínica Universitária de Doenças Infecciosas, Faculdade de Medicina, Universidade de Lisboa, Lisbon, Portugal. ${ }^{4}$ Serviço de Infecciologia, Hospital de S. Bernardo, Setúbal, Portugal and ${ }^{5}$ Serviço de Infecciologia, Hospital Fernando da Fonseca, Amadora, Portugal. Correspondence: AE Sousa (asousa@medicina.ulisboa.pt)

${ }^{6}$ Present address: Instituto Gulbenkian de Ciência, Oeiras, Portugal.

${ }^{7}$ Present address: Antibody and Vaccine Group, Cancer Sciences Unit, University of Southampton, Faculty of Medicine, University Hospital Southampton, UK.

${ }^{8}$ These authors are joint senior authors. 
that persists throughout the chronic phase, with antiretroviral therapy (ART) failing to consistently recover this deficit. ${ }^{9,10}$ Moreover, this lack of immunological reconstitution at the mucosal level is linked to persistent inflammation and comorbidities in long-term-treated HIV-1-infected individuals. ${ }^{11}$

The loss of mucosal CD4 T-cells able to produce IL-17 (Th17) and/or IL-22 (Th22) is particularly marked, and is paralleled by a relative expansion of Foxp3-expressing Tregs. ${ }^{12}$ Although, CD4 T-cell loss has been attributed to the direct cytopathic effects of the virus, other mechanisms are also likely to be involved such as alterations in T-cell traffic and mucosal recruitment, as well as collagen deposition leading to architectural alterations. ${ }^{13,14}$ Furthermore, recent reports emphasize the concomitant loss of ILC3, which are not direct targets of HIV/SIV infection, ${ }^{15}$ as well as epithelial leakage, leading to gut microenvironment disruption. ${ }^{16}$ In agreement with an important role for ILC3, we have recently shown that a preserved ILC3 compartment was associated with mucosal integrity in long-term-treated HIV-1 individuals with persistent CD4 T-cell depletion. ${ }^{17}$

The strategies utilized by the gut to cope with persistent viral infection remain undefined. In order to address this, we investigated gut homeostasis in the context of chronic HIV-2 infection, which is characterized by reduced mortality in the absence of ART. ${ }^{18}$ HIV-2-infected individuals feature a progressive CD4 T-cell depletion that directly correlates with markers of immune activation and systemic inflammation as observed in HIV-1 infection, but with a much slower rate of progression to AIDS. ${ }^{19,20}$ Importantly, HIV-2 targets the same cell populations as HIV-1, and is able to establish a disseminated infection with evidence of persistent ongoing viral replication despite the low to undetectable viremia observed even in the absence of ART. $^{21,22}$ The mechanisms underlying this relatively benign course are incompletely understood, but are unlikely to result from intrinsic viral properties, ${ }^{23}$ and may be in part due to the host-specific immune responses. ${ }^{24}$

As yet, no direct studies of gut mucosa in HIV-2-infected individuals have been reported. A previous study conducted in an African setting showed increased plasma lipopolysaccharide levels, suggesting that gut leakage and microbial translocation occurs in HIV-2 infection. ${ }^{25}$ We have compared Europeanresident HIV-2- and HIV-1-infected individuals and found that, in contrast to HIV-1, there was no significant increase in lipopolysaccharide levels in HIV-2 infection despite the upregulation of markers of $\mathrm{T}$-cell and monocyte activation in both infections. ${ }^{26}$

\section{Table 1 Cohort characterization}

\begin{tabular}{|c|c|c|c|c|}
\hline & Seronegatives & HIV-2 & Untreated HIV-2 & Treated HIV-2 ${ }^{\mathrm{a}}$ \\
\hline Age & $58(49-63)$ & $61(58-65)$ & $62(58-66)$ & $59(58-64)$ \\
\hline Viremia (RNA copies per ml) & NA & $<40$ & $<40$ & $<40$ \\
\hline CD4 T-cells per $\mu$ l & $671(575-781)$ & $489(301-734)$ & $670(490-777)$ & 407 (253-613) \\
\hline \multicolumn{5}{|l|}{$\%$ Naive $\left(C D 45 R O^{-} C C R 7^{+}\right)^{\mathrm{C}}$} \\
\hline Within CD4 T-cells & $30.5(25.8-41.5)$ & $43.7(33.9-49.3)$ & $43.0(42.1-47.2)$ & $44.3(22.6-52.4)$ \\
\hline Within CD8 T-cells & $16.8(3.8-31.7)$ & $10.9(7.0-19.2)$ & $10.9(5.7-14.9)$ & $10.9(10.7-20.6)$ \\
\hline \multicolumn{5}{|l|}{$\% H L A-D R^{+} C D 38^{+c}$} \\
\hline$\% \mathrm{CD} 16^{+}$within $\mathrm{CD} 14^{\text {bright }}$ monocytes $^{\mathrm{c}}$ & $4.2(2.8-5.6)$ & $6.6(4.3-13.5)^{\mathrm{b}}$ & $13.5(6.3-14.5)^{b}$ & $6.6(4.3-13.5)$ \\
\hline \multicolumn{5}{|l|}{ Memory-effector CD4 T-cells ${ }^{c}$} \\
\hline$\% \mathrm{IL}-22^{+}$ & $1.4(1.0-2.8)$ & $0.9(0.7-1.9)$ & $0.9(0.6-3.4)$ & $0.8(0.7-1.2)$ \\
\hline$\% \mathrm{IL}-17^{+}$ & $1.1(0.8-2.1)$ & $1.2(0.9-1.5)$ & $1.2(0.8-1.7)$ & $1.2(0.9-1.5)$ \\
\hline$\% \mathrm{IFN} \gamma^{+}$ & $9.1(5.1-39.6)$ & $15.6(11.2-20.3)$ & $15.7(12.7-29.4)$ & $12.4(4.3-20.7)$ \\
\hline
\end{tabular}


Therefore, we reasoned that the investigation of gut mucosa in HIV-2 infection could reveal mechanisms able to counteract the impact of persistent injury, such as that caused by HIV.

We found evidence of HIV-2 replication in both sigmoid and ileal biopsies from HIV-2-infected individuals irrespective of ART. Notwithstanding this, absolute numbers of CD4 T-cells, including Th17/Th22 and FOXP3 + Tregs, as well as of ILC3, were preserved in the lamina propria. T-cell preservation was associated with increased expression of granulocyte-macrophage colony-stimulating factor (GM-CSF) that directly correlated with levels of chemokines involved in Th17 and Th22 recruitment, CCL20 and CCL28, respectively. Importantly, epithelial tight junctions were not disrupted and in vitro data confirmed the lack of impact of HIV-2 isolates on the expression of the tight junction protein zonula occludens (ZO-1). Overall, our data support a model in which the inability of HIV-2 to perturb epithelial integrity allows for the development of mechanisms able to maintain mucosal homeostasis in the context of viral replication.

\section{RESULTS}

\section{Evidence of HIV-2 replication in the gut mucosa}

We first asked whether HIV-2 infects and replicates in the gut mucosa. Sigmoid and ileum biopsies were collected during routine colon cancer screening from 13 HIV-2-infected individuals, featuring undetectable viremia even in the absence of ART (Table 1). HIV-2 patients featured a broad distribution of circulating CD4 T-cell counts. Of note, there were no significant differences between treated and untreated HIV-2infected patients, except for lower levels of CD8 T-cells and monocyte activation markers in treated individuals (Table $\mathbf{1}$ ). In agreement with previous reports, ${ }^{21,27}$ ART was not associated with significant increase of circulating CD4 T-cell levels or changes in CD4 T-cell activation markers (Table 1). We quantified cell-associated HIV-2 DNA in sigmoid and ileum biopsies, and found that 8/11 ileum and 11/12 sigmoid biopsies assessed were positive, featuring comparable levels (Figure 1a, $P=0.27$ ). Of note, cell-associated DNA levels were significantly higher in sigmoid than in peripheral blood mononuclear cells $(\mathrm{PBMC})(P=0.04)$. We further confirmed HIV-2 mucosal replication via immunohistochemistry using a previously validated anti-HIV-2 Gag-specific antibody, ${ }^{22}$ using one biopsy per patient. Cells positive for HIV-2 Gag protein were detected in both sigmoid and ileum biopsies irrespective of antiretroviral treatment (sigmoid: $2 / 6$ untreated and 3/7 ART patients; ileum: 1/5 untreated and 3/7 ART patients, Figure 1b).

Overall, our results reveal for the first time that HIV-2 infects and continuously replicates in the gut mucosa, despite the studied HIV-2-infected patients featuring undetectable viremia and irrespectively of ART.

\section{CD4 T-cells are not depleted in the gut of HIV-2-infected patients}

Next, we asked whether ongoing viral replication was associated with mucosal CD4 T-cell depletion in HIV-2-infected
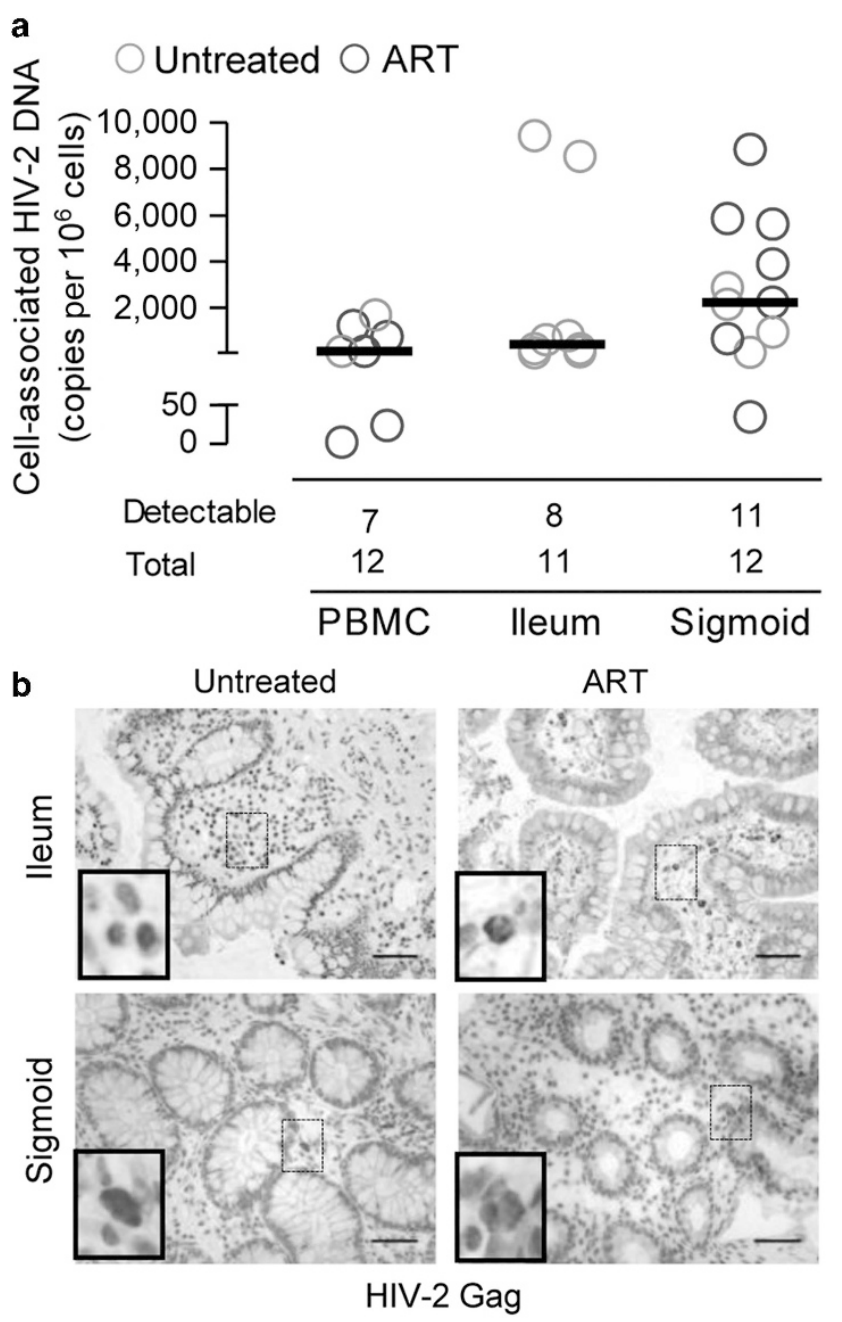

Figure 1 Evidence of viral replication in sigmoid and ileum mucosa of HIV-2-infected individuals. (a) Levels of cell-associated HIV-2 DNA in peripheral blood, ileum, and sigmoid samples of HIV-2-infected individuals. Each symbol represents one individual and only those with detectable levels are shown; bar indicates median; numbers below the graph indicate the total number of individual samples and those with levels above the detection threshold of the assay. Two-group comparisons were done using the Mann-Whitney $U$-test; $P$-values $<0.05$ are shown. (b) Representative immunohistochemistry images of Gag (p27) staining of ileum and sigmoid biopsy sections from untreated and ART HIV-2-infected individuals (bars $=50 \mu \mathrm{m}$ ). A digitally amplified image showing positive cells is shown in the lower left corner. ART, antiretroviral therapy.

individuals, as observed in HIV-1 and SIV infections. ${ }^{9}$ We found no differences between HIV-2-infected and seronegative individuals in terms of the number of lamina propria $\mathrm{CD} 4 \mathrm{~T}$-cells in ileum (Figure 2a, $P=0.47$ ) and sigmoid (Figure 2b, $P=0.27$ ) biopsies, quantified by immunohistochemistry, or in the proportion of CD4 T-cells within total sigmoid lymphocytes, assessed in biopsy-derived cell suspensions by flow cytometry (Figure 2c, $P=0.27$ ).

In agreement with the presence of mucosal viral replication we found increased proportions of CD8 T-cells in sigmoid mucosa (Figure 2c, $P=0.04$ ), as well as significant expansion of those able to produce interferon- $\gamma($ IFN- $\gamma$ ) (Figure 2c, 
a $\quad$ lleum

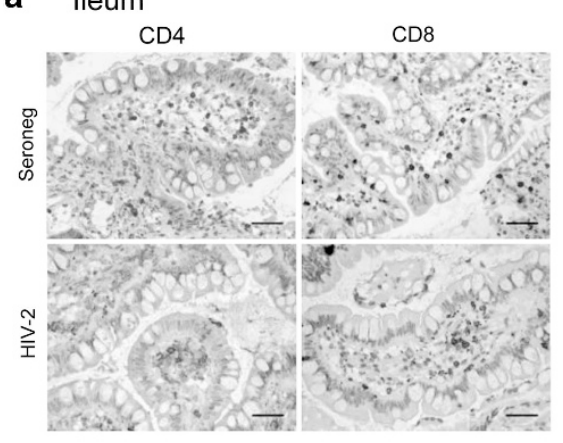

b Sigmoid

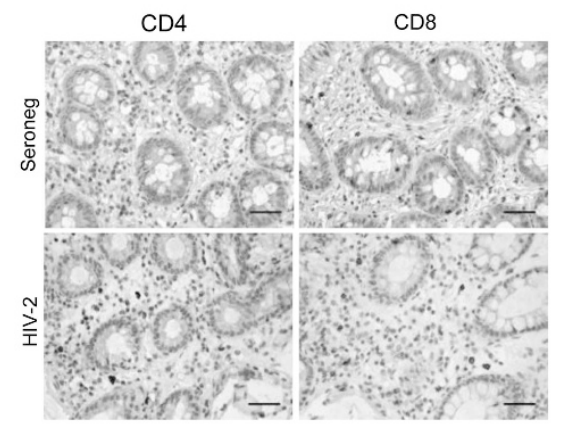

Untreated $O A R T$

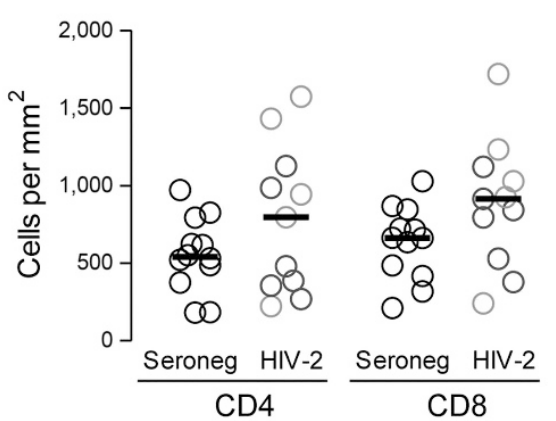

Untreated OART

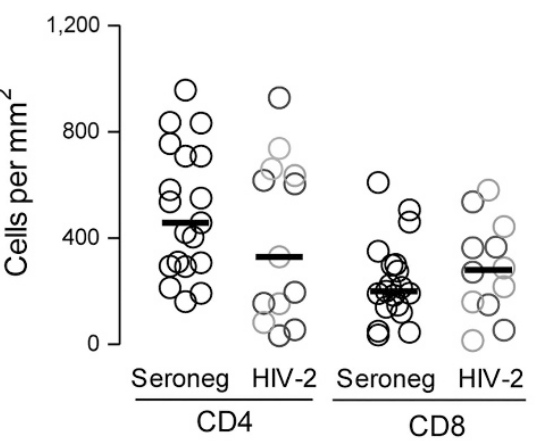

c

\section{Sigmoid CD3}

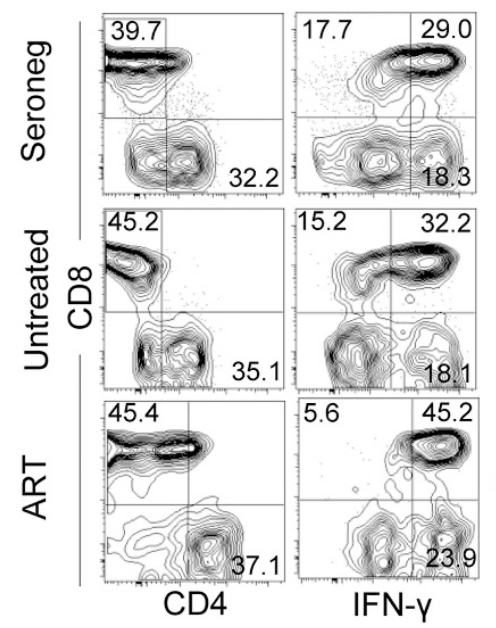

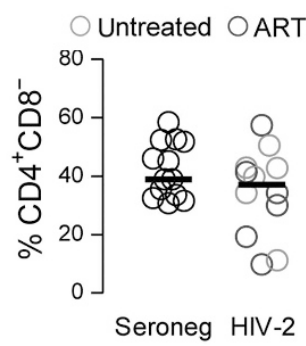

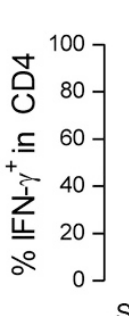

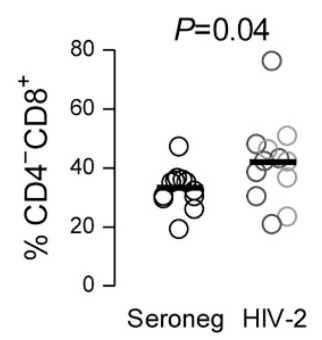

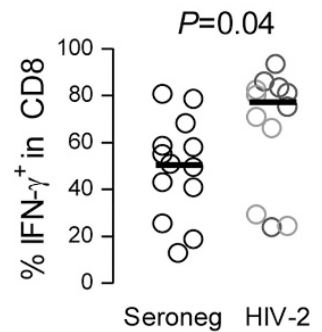

Figure 2 T-cell subsets in the gut mucosa of HIV-2-infected individuals. (a-b) Representative images of CD4 and CD8 immunohistochemistry staining of ileum (a) and sigmoid (b) biopsy sections from seronegative and HIV-2-infected individuals (bars = $50 \mu \mathrm{m}$ ); graphs show CD4 and CD8 cells per mm ${ }^{2}$ in biopsies obtained from both cohorts. (c) Representative dot plots of CD8/CD4 and CD8/IFN- $\gamma$ staining within a CD3 + gate, in cell suspensions obtained from sigmoid biopsies of seronegative and HIV-2-infected (untreated and ART) individuals; graphs show the relative proportion of CD4 + CD8 - (upper left hand graph), and CD4-CD8 + cells (upper right hand graph) within a CD3 + gate, as well as the frequency of IFN $\gamma$-producing cells within CD4 (lower left hand graph) and CD8 T-cells (lower right hand graph) in the two cohorts. Each symbol represents one individual and bar indicates median. Two-group comparisons were done using the Mann-Whitney $U$-test; $P$-values $<0.05$ are shown. IFN, interferon.

$P=0.04)$. Conversely, the frequency of IFN- $\gamma$-producing CD4 T-cells was similar in HIV-2-infected and seronegative individuals (Figure 2c, $P=0.29$ ).

Of note, untreated and treated HIV-2-infected individuals featured no differences in sigmoid CD4 cells $/ \mathrm{mm}^{2}$ (untreated: 483 (136-679, $n=6)$; ART: 196 (55-617, $n=7) ; P=0.53)$, or CD8 cells per $\mathrm{mm}^{2}$ (untreated: $254(125-479, n=6)$; ART: 319
(127-411, $n=6) ; P=1.00)$. Additionally, CD4 T-cell counts did not differ in those patients with detectable Gag protein as compared to those without (multiple linear regression controlling for ART and presence of p27, $P>0.38$ ), nor was there a correlation between the number or frequency of CD4 T-cells in the sigmoid mucosa and peripheral blood $(r=0.31, P=0.31$, and $r=-0.01, P=0.97$, respectively). Similarly, no 
differences in ileum CD4 cells per $\mathrm{mm}^{2}$ (untreated: 946 (5101,503, $n=5)$; ART: 434 (335-1,021, $n=6) ; P=0.43)$ or CD8 cells per $\mathrm{mm}^{2}$ (untreated: $771(437-1,107, n=5)$; ART: 614 $(367-725, n=6) ; P=0.25)$ were seen in untreated and treated HIV-2-infected individuals, and again no correlation between ileum and peripheral blood CD4 numbers was observed $(r=-0.06 ; P=0.86)$.

We also found no association between sigmoid CD4 T-cell numbers and the frequency of HLA-DR $+\mathrm{CD} 38+$ cells within circulating CD4 and CD8 T-cell subsets $(r=-0.39 ; P=0.21$ and $r=-0.08 ; P=0.80$, respectively), or with the proportion of CD16 bright monocytes $(r=-0.32 ; P=0.34)$. Thus, in HIV-2-infected individuals, the significant increase in activation markers within circulating CD4 and CD8 T-cells, as well as monocytes (Table 1), was not linked to depletion of mucosal CD4 T-cells.

In summary, gut mucosal CD4 $\mathrm{T}$-cells were preserved during chronic HIV-2 infection despite the evidence of ongoing viral replication at this site.

\section{Preserved mucosal Th17/Treg balance during HIV-2 infection}

The functional characterization of the mucosal CD4 T-cell compartment revealed that IL-17-producing CD4 T-cells were preserved, with no difference in their frequency in cell suspensions generated from sigmoid biopsies of HIV-2 and seronegative individuals (Figure 3a, $P=0.66$ ). Furthermore, absolute numbers of IL-17 + cells per $\mathrm{mm}^{2}$ in sigmoid (Figure $3 \mathbf{b}, P=0.68$ ) and ileum biopsy sections (Figure $3 \mathbf{c}, P=0.66$ ) determined by immunohistochemistry were also similar in both cohorts.

Additionally, HIV-2-infected individuals featured no expansion of the Treg compartment. FOXP3 + Treg frequency in cell suspensions from sigmoid biopsies, assessed by flow cytometry (Figure 3a, $P=0.46$ ), and numbers of FOXP3 + cells per $\mathrm{mm}^{2}$, assessed by immunohistochemistry in sigmoid and ileum biopsy sections (Figure $3 \mathbf{b}, P=0.97$; and Figure $3 \mathbf{c}, P=0.83$; respectively) were similar to those observed in the seronegative cohort.

The balance between these two populations is considered particularly relevant for maintaining mucosal homeostasis. ${ }^{28,29}$ This was also unaltered in HIV-2 infection (IL-17 + /FOXP3 + cell counts in sigmoid: $1.99(0.29-3.98, n=12)$ vs. $0.69(0.27-$ $1.98, n=14), P=0.14$; and ileum $1.23(0-4.62, n=11)$ vs. 2.48 (0.90-5.87, $n=11), P=0.27$; in HIV-2-infected and control individuals, respectively).

Overall, the lack of mucosal CD4 T-cell depletion is also accompanied by a preserved balance between Th17 and FOXP3 + cells in HIV-2 infection.

\section{IL-22-dependent mucosal homeostasis is preserved in HIV-2 infection}

IL-22 is fundamental for maintaining gut homeostasis, as it facilitates a unique cross-talk between immune cells and epithelia, particularly in persistent inflammation. ${ }^{5}$ We first assessed whether Th22 were preserved in HIV-2-infected individuals. The distribution within total CD4 T-cells of the subsets able to produce one, two, or the three cytokines (IL-17, IL-22, and IFN $\gamma$ ), was similar in HIV-2-infected and healthy individuals (Figure 4, $P=0.13$ ). Th22 frequency in sigmoidderived cell suspensions, as well as the number of sigmoid and ileum IL- $22+\mathrm{ROR} \gamma+\mathrm{CD} 3+$ cells per $\mathrm{mm}^{2}$, evaluated by immunofluorescence, did not differ in HIV-2-infected and seronegative individuals (Figure 5a, $P=0.98$; Figure $5 \mathbf{b}$ top, $P=0.85$; Figure $5 \mathrm{c}$ top, $P=0.93$; respectively). Of note, we have previously shown that this population remained depleted in long-term-treated HIV-1-infected individuals. Sigmoid CD4 $\mathrm{T}$-cell quality was further assessed by flow cytometry, in terms of concomitant production of IL-22 and IL-17. We observed no differences with respect to the frequency of $\mathrm{CD} 4$ T-cells producing IL-17 alone $(3.8(2.7-8.3) \%$ vs. 3.6 (3.05.7)\%; $P=0.95)$, IL-22 alone $(2.9(1.8-4.7) \%$ vs. $2.8(2.1-5.3) \%$; $P=0.84)$, or both cytokines $(3.0(1.5-4.2) \%$ vs. $2.8(2.0-6.1) \%$; $P=0.37)$ in HIV-2-infected and seronegative individuals, respectively. Regarding ILC3, defined here as cKit + Nkp44 + cells lacking lineage markers, ${ }^{30}$ we found similar frequencies within total CD45 + cells in both cohorts (Figure 4d, $P=0.41$ ). Additionally, as ILC3 constitute the major non-T-cell source of IL-22, ${ }^{4}$ we quantified the frequency of IL-22-producing cells within a CD3 - gate and found no differences between HIV-2-infected and seronegatives individuals (6.1 (2.9-7.5)\% vs. $2.6(0.8-9.0) \%$, respectively; $P=0.13)$. As ILC3 can also produce IL-17, ${ }^{4}$ it is also worth noting that the frequency of IL-17-producing CD3 - cells was similar (2.5 (2.1-3.5) vs. 2.6 (0.8-9.0) in HIV-2-infected and seronegative individuals, respectively; $P=0.14$ ). Furthermore, we assessed the numbers of ILC3, defined as CD3 - ROR $\gamma+$ IL-22 + cells, in sigmoid and ileum biopsy sections by immunofluorescence (Figure 5b bottom, $P=0.62$; Figure 5c bottom, $P=0.65$, respectively), and found no inter-cohort differences.

In order to evaluate the functional implications of these findings, we quantified the expression levels of mucins and antimicrobial peptides regulated by IL-22 signaling. Bulk mucin production was evaluated by periodic acid Shiff-Alcien Blue staining, and found to be similar in sigmoid and ileum biopsies of seronegative and HIV-2-infected individuals (Figure $5 \mathbf{e}$ and $\mathbf{f}$, respectively). Furthermore, we found no inter-cohort differences in mucin and antimicrobial peptide gene expression levels in either sigmoid or ileum (Figure 5e and $\mathbf{f}$, respectively; $P>0.07$ for all genes) biopsies, with the exception of increased expression of mucin 13 in the ileum of HIV-2-infected individuals $(P<0.001)$.

Overall, sigmoid and ileum IL-22 production appear to be unaffected in HIV-2-infected individuals, a situation reflected by their maintained expression of markers of IL-22-dependent mucosal homeostasis.

\section{Preserved tight junctions in HIV-2 infection}

We hypothesized that maintained mucosal T-cell homeostasis might result from an intact epithelial barrier in the context of chronic HIV-2 infection. To test this possibility, we quantified the levels of ZO-1 protein, an important tight junction component, by immunofluorescence in different gut epithelial 
a

Sigmoid CD4

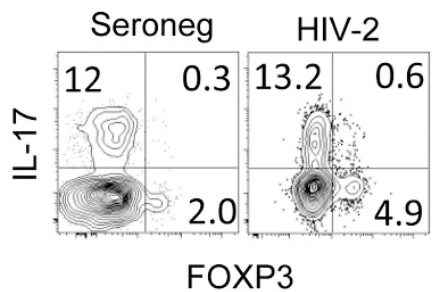

b

Sigmoid

Seroneg

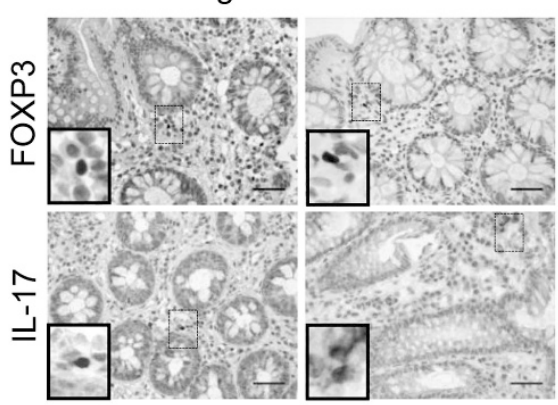

c lleum

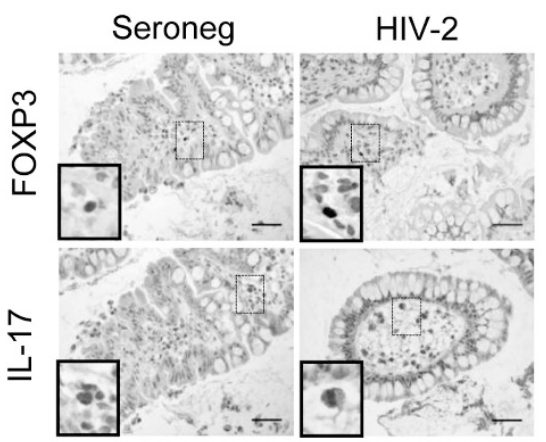

Untreated OART
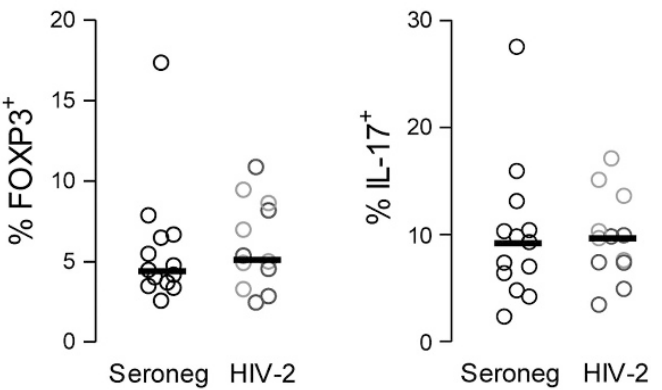
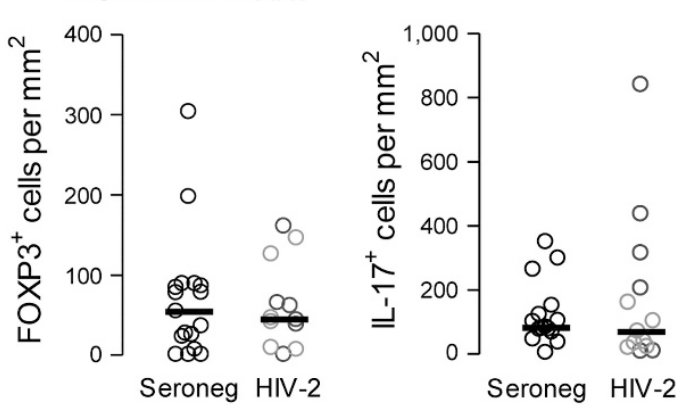

0
0
0
5
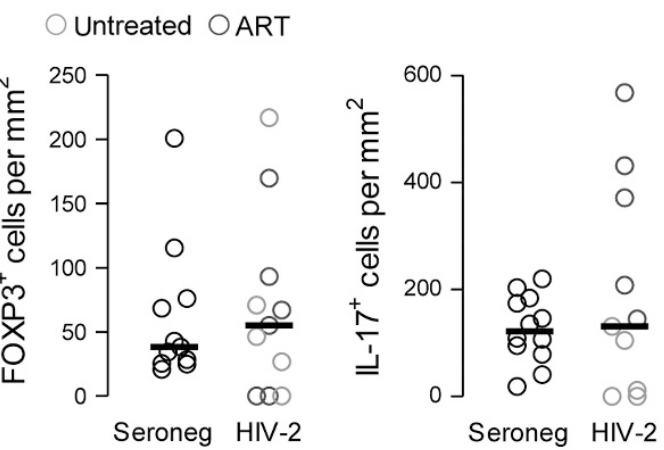

Figure 3 Preserved Th17 and regulatory CD4 T-cell subsets in the gut mucosa of HIV-2-infected individuals. (a) Representative dot plots of IL-17 and FOXP3 staining within a CD4 T-cell gate in cell suspensions obtained from sigmoid biopsies of HIV-2-infected and seronegative individuals. Graphs show the frequency of FOXP3 + (left hand graph), and IL-17 + (right hand graph) cells within total sigmoid CD4 T-cells for each cohort. (b-c) Representative images of FOXP3 and IL-17 immunohistochemistry staining in sections of a sigmoid (b) and ileum (c) biopsies from seronegative and HIV-2-infected individuals (bars $=50 \mu \mathrm{m}$ ) with a digitally amplified image of positive cells shown in the lower left corner; graphs show the absolute number of FOXP3 + (left hand graph) and IL-17+ (right hand graph) cells per $\mathrm{mm}^{2}$ for each cohort. Each symbol represents one individual and bar indicates median. Two-group comparisons were done using the Mann--Whitney $U$-test; $P$-values $<0.05$ are shown. IL, interleukin.

regions. We found similar $\mathrm{ZO}-1$ expression in both the villi and upper epithelium of ileum (Figure 6a, $P=0.14$ ), as well as the upper epithelium and crypts in the colon (Figure $6 \mathbf{b}, P=0.97$ ), in both cohorts. Of note, ZO-1 protein levels did not correlate with any of the above evaluated parameters, including IL-22 and the expression levels of IL-22-inducible genes.

Furthermore, we investigated the direct impact of HIV-2 on tight junction integrity, using an in vitro gut epithelial cell line model, with ZO-1 protein expression assessed by immunofluorescence as a read-out. ${ }^{31}$ Both HIV-2 lab-adapted and primary isolates, utilizing either CXCR4 or CCR5 co-receptors, showed a reduced capacity to disrupt the ZO-1 network as illustrated in Figure $6 \mathrm{c}$, as compared to $\mathrm{HIV}-1_{\text {IIIB }}$, used here as a positive control. ${ }^{31}$

Overall, these data suggest that gut mucosal integrity, at the level of epithelial tight junctions, is maintained during chronic HIV-2 infection, likely as a result of the reduced ability of HIV-2 to directly perturb the tight junction structure.

\section{Increased mucosal expression levels of GM-CSF and} chemokines linked to T-cell recruitment in HIV-2 infection Finally, we asked whether the lack of tight junction disruption was effectively linked to preservation of the mucosal microenvironment despite HIV-2 replication. With this in mind, we 


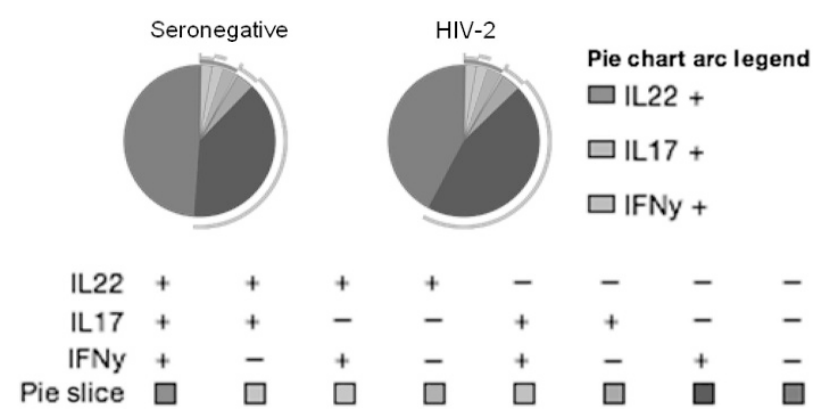

Figure 4 Preserved distribution of cytokine production by mucosal CD4 T-cell subsets in HIV-2-infected individuals. The distribution of IL-17, IL-22, and IFN $\gamma$ production within sigmoid CD4 T-cells was analyzed using SPICE software and no differences were found using partial permutation test. IFN, interferon; IL, interleukin.

assessed the degree of structural disruption and collagen deposition by immunohistochemistry using Masson's trichrome, and quantified the transcriptional levels of a panel of genes involved in local inflammatory processes and cell homeostasis.

We found a relatively preserved structure of both ileum and sigmoid mucosa in HIV-2-infected individuals, as illustrated in Figure $7 \mathbf{a}$ and $\mathbf{b}$, respectively. A blinded assessment by a trained pathologist revealed no significant difference in cellular infiltrate, as well absence of epithelial erosions and follicular structures within both ileum and sigmoid lamina propria (neutrophils, lymphocytes, and eosinophils), resulting in a comparable inflammatory score to seronegatives (sigmoid: $1.0(1.0-2.0, n=13)$ vs. $1.0(1.0-1.5, n=15), P=0.50$; and ileum $1.0(1.0-1.0, n=9)$ vs. $1.0(1.0-1.0, n=11), P=0.22$; in HIV-2-infected and seronegative individuals, respectively). Additionally, we found no evidence of increased collagen deposition in either ileum or sigmoid biopsies (Figure $7 \mathbf{a}$ and $\mathbf{b}$, respectively), except in two untreated HIV-2-infected patients where there was increased collagen deposition in the ileum in the absence of detectable Gag protein or $\mathrm{CD} 4+$ cell depletion $\left(1,431\right.$ and 223 cells per $\left.\mathrm{mm}^{2}\right)$.

In agreement, both cohorts featured comparable gene expression levels of cytokines and other molecules known to be involved in the differentiation and/or survival of Th17, Th22, and Treg CD4 subsets, namely IL-6, IL-23, TGF $\beta$, and IDO1 (Figure $7 \mathbf{a}$ and $\mathbf{b} ; P>0.55$ for all genes). Of note, sigmoid biopsies from HIV-2-infected individuals featured lower levels of AHR (Figure 7b, $P=0.004$ ), a receptor relevant for the survival, proliferation, and function of both ILC3 and Th22. ${ }^{32,33}$ The relative amounts of the key anti- and pro-inflammatory cytokines, IL-10 and IL-1 $\beta$ were similar in both cohorts (Figure 7a and $\mathbf{b} ; P>0.76$ for both). Moreover, in spite of the evidence of local HIV-2 replication, there was no increase in the transcriptional levels of $M x A$ (Figure 7a for ileum and Figure $\mathbf{7 b}$ for sigmoid), a type I interferon inducible gene. $^{26}$

In this context, it is worth noting that GM-CSF expression was increased in the sigmoid of HIV-2-infected individuals (Figure $7 \mathbf{b}, P=0.04$ ).
We also quantified the expression of two chemokines implicated in the recruitment of T-cells to the gut mucosa: CCL20, whose receptor, CCR6, is expressed by Th17 cells, ${ }^{34}$ and CCL28, whose receptor, CCR10, is expressed on Th22 cells. ${ }^{35}$ Importantly, we found a significant increase in CCL20 and CCL28 expression in sigmoid biopsies of HIV-2-infected individuals (Figure 7c, $P=0.007$ and $P=0.001$, respectively), suggesting that increased cell recruitment may play a role in maintaining CD4 T-cells in the sigmoid lamina propria of these individuals. Moreover, there was a direct correlation between the levels of GM-CSF and both CCL20 and CCL28 (Figure 7c), suggesting that GM-CSF may help orchestrate this chemokine response. Of note, although we did not find increased gene expression levels of these chemokines in the ileum (CCL20: $1,749(1,054-3,344)$ vs. $1,954(1,382-19,014), P=0.38$; CCL28: $1,491(1,106-2,234)$ vs. $2,078(1,332-5,871), P=0.15$; arbitrary units, 11 HIV-2 vs. 10 seronegative individuals), the correlations between GM-CSF and CCL20 $(r=0.5$, $P=0.01)$ and CCL28 $(r=0.66, P<0.001)$ were maintained.

In conclusion, HIV-2-infected individuals featured a normal gut mucosal structure without evidence of local inflammation, paralleled by increased gene expression levels in sigmoid biopsies of GM-CSF and chemokines involved in CD4 T-cell recruitment, which likely help maintain the CD4 T-cell compartment and overall gut mucosal homeostasis. Our data led us to hypothesize that this local response, able to counteract any potential $\mathrm{CD} 4$ depletion induced by ongoing viral replication, may result from the absence of disruption of epithelial tight junctions and the consequent preservation of epithelial integrity in the context of HIV-2 infection.

\section{DISCUSSION}

This first study of chronic HIV-2-infected individuals' gut mucosa provides evidence for: (a) viral replication in the ileum and sigmoid, despite the undetectable viremia observed in all patients; (b) lack of CD4 T-cell depletion and maintained Th17 and Th2 2 compartments in the absence of increased FOXP3 + Tregs; (c) preserved ILC3 cells, and innate production of IL-22 and IL-17; (d) normal mucosal structure and epithelial integrity; (e) preserved tight junctions in vivo and in an in vitro model of HIV-2 infection; and (f) increase in GM-CSF production in direct association with increased levels of chemokines involved in Th17 and Th22 recruitment, CCL20 and CCL28, respectively.

Our data support a model in which the maintenance of epithelial tight junctions and overall mucosal integrity, despite local HIV replication, protects mucosa from overwhelming microbial stimuli, and, thus, allows the establishment of repair mechanisms and mucosal responses to counteract CD4 T-cell depletion by promoting mucosal $\mathrm{T}$-cell recruitment.

Our finding of ongoing HIV-2 replication in the gut mucosa despite undetectable levels of plasma viral load supports a compartmentalization of HIV-2 replication in the gut-associated lymphoid tissue. The mechanisms underlying the tissue control of HIV-2 viral replication, yet to be deciphered, can provide valuable information for understanding the control of 
a
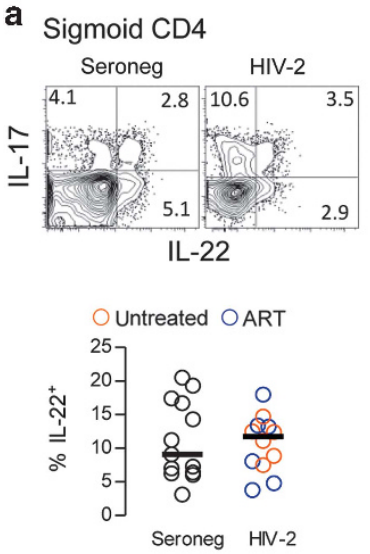

c lleum
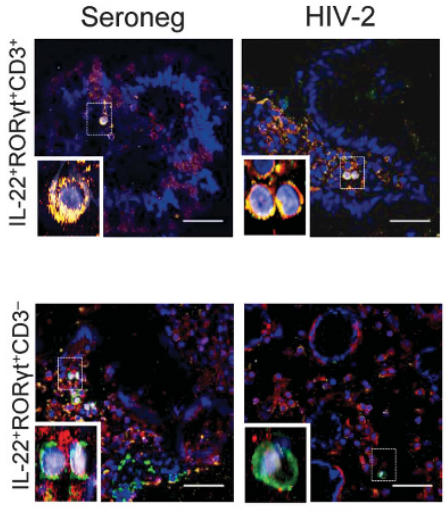

DAPI IL-22 RORyt CD3

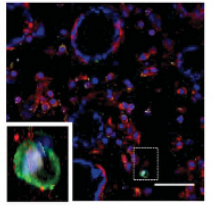

b Sigmoid
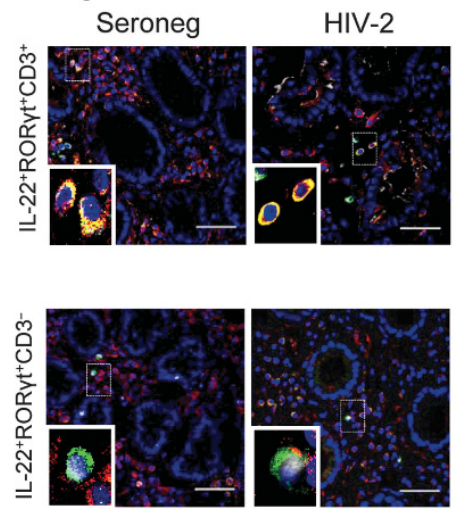

DAPI IL-22 RORyt CD3
Untreated OART

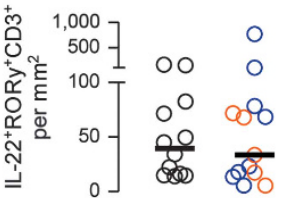

Seroneg HIV-2

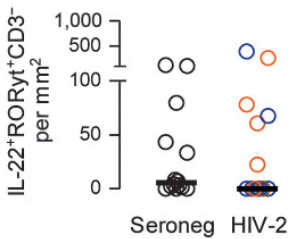

d Sigmoid CD45

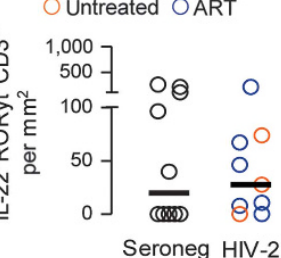

Seroneg HIV-2

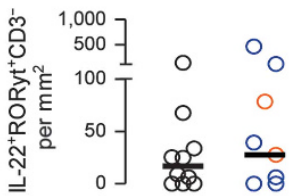

Seroneg HIV-2
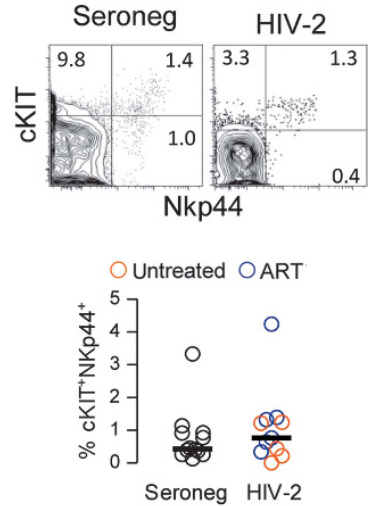

e

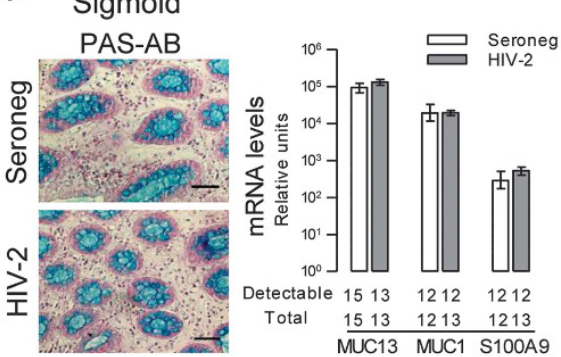

f

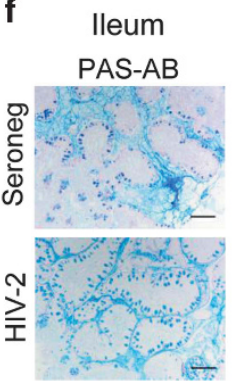

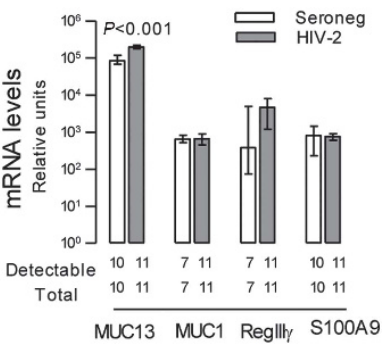

Figure 5 Preserved IL-22 production and expression of IL-22-associated genes in the gut of HIV-2-infected individuals. (a) Representative dot plots of IL-17 and IL-22 staining within a CD4 T-cell gate, in cells isolated from sigmoid biopsies of seronegative and HIV-2-infected individuals; graph shows the frequency of IL-22 + cells within total sigmoid CD4 T-cells for each cohort. (b) Representative images of immunofluorescence staining for IL-22, ROR $\gamma \mathrm{t}$, and CD3 in sections of a sigmoid biopsy from a seronegative and HIV-2-infected individual (bars $=50 \mu \mathrm{m})$. The lower left corner features a digitally amplified image of triple-positive (ROR $\gamma \mathrm{t}+\mathrm{IL}-22+\mathrm{CD} 3+)$ and double-positive (ROR $\gamma \mathrm{t}+\mathrm{IL}-22+\mathrm{CD} 3-$ ) cells in the upper and lower panels, respectively. Graphs show the number of triple (top) and double (bottom) positive cells per $\mathrm{mm}^{2}$ for each cohort. (c) The same as (b) in ileum biopsies. (d) Representative dot plot of cKit/NKp44 staining within a CD45 + lineage-gate for a seronegative and an untreated HIV-2-infected individual; graph shows the frequency of cKIT + NKp44 + within CD45 + /lineage-cells for each cohort. (e-f) Representative PAS-AB staining in sections from sigmoid (e) and ileum (f) biopsies from seronegative and HIV-2-infected and individuals (bars $=50 \mu \mathrm{m}$ ). Bar graphs show the median and interquartile range of $\mathrm{mRNA}$ levels of IL-22-inducible genes (mucins and antimicrobial molecules) in biopsies from seronegative and HIV-2-infected individuals; numbers below the graph indicate the total number of samples tested and those with levels above the detection threshold of the respective gene. Results are expressed in relative units, normalized to the mean cycle threshold $(\mathrm{Ct})$ levels of GAPDH and r18S. Each symbol in the scatter graphs represents an individual and bars indicate median. Two-group comparisons were made using the Mann-Whitney U-test; $P$-values $<0.05$ are shown. IL, interleukin; PAS-AB, periodic acid Shiff-Alcien Blue.

HIV replication and reservoirs in HIV-1-infected individuals under ART. We and others have shown that the levels of cellassociated proviral DNA in PBMC are comparable in HIV-1 and HIV-2 infections, ${ }^{21,23}$ supporting an equal ability of the two viruses to disseminate and persist. CCR5, expressed by the majority of mucosal CD4 T-cells, has been suggested to be the 
a

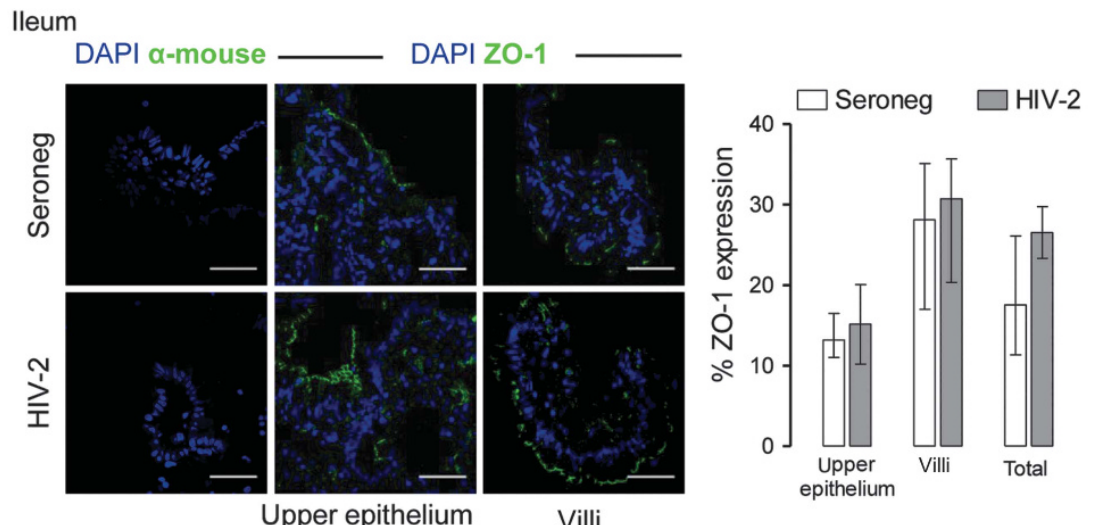

b Sigmoid
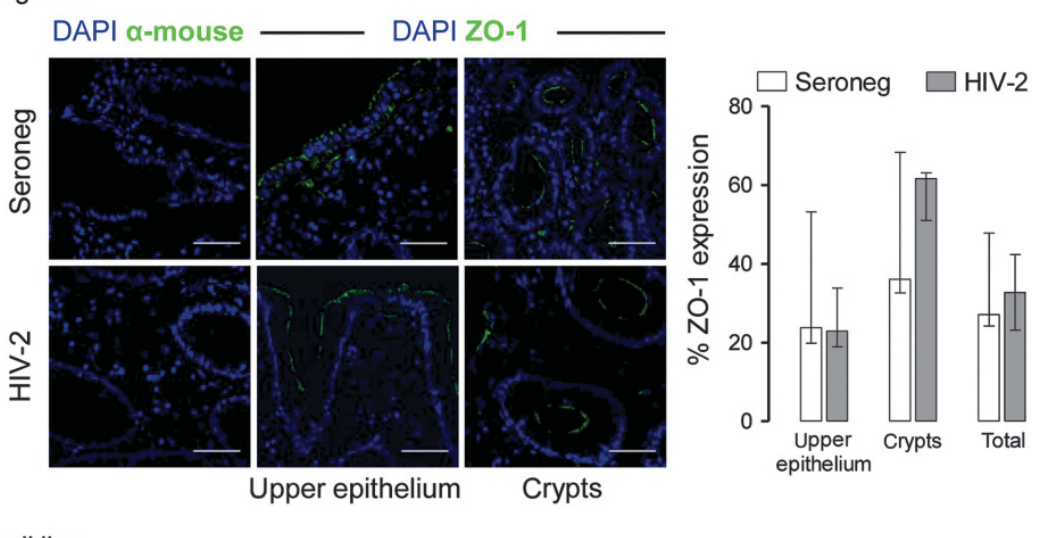

C Colon cell line

DAPI a-mouse
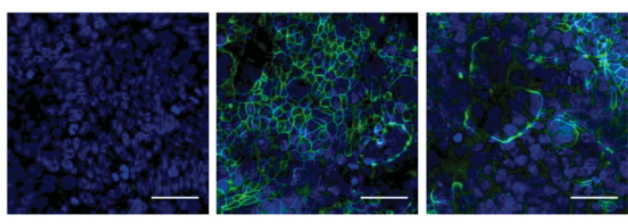

DAPI ZO-1

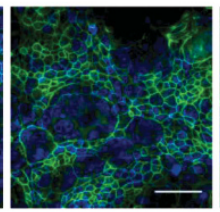

HIV-2 ${ }_{\text {ROD }}$

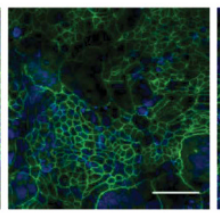

HIV-2 R5

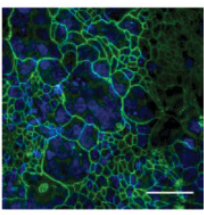

HIV-2 X4

Medium alone

Lab-adapted X4 viruses

Primary viral isolates

Figure 6 Lack of tight junction disruption in HIV-2 infection. (a-b) Representative images of ZO-1 immunofluorescence staining in sections of an ileum (a) and a sigmoid (b) biopsy from seronegative and HIV-2-infected individuals (bars $=50 \mu \mathrm{m}$ ), showing staining control (secondary antibody only, left hand panels), upper epithelium (central panels) and villi (a) or crypts (b) (right hand panels). Bar graphs show median and interquartile range of the percentage of ZO-1 expression in upper epithelium, villi, or crypts, and overall, in ileum (a) and sigmoid (b) biopsies from HIV-2 ( $n=13$ and 10 , respectively) and seronegative ( $n=5$ and 8, respectively) cohorts. Two-group comparisons were made using the Mann-Whitney $U$-test, and no significant differences were found. (c) ZO-1 immunofluorescence staining of T84 cells cultured in medium alone or with the lab-adapted X4 viruses HIV-2 ${ }_{\text {ROD }}$ or HIV-1 $1_{\text {IIIB }}$, as well as the X4 (HIV-2 $\left.2_{20.04}\right)$ or R5 $\left(\mathrm{HIV}-2_{60,415} \mathrm{~K}\right) \mathrm{HIV}-2$ primary isolates $(\mathrm{bars}=50 \mu \mathrm{m})$; representative images of three independent experiments are shown. ZO-1, zonula occludens.

main in vivo co-receptor for HIV-2, ${ }^{36}$ which is further supported by our data revealing the preferential targeting of the gut mucosa. In all five patients with undetectable proviral DNA in PBMC, there were detectable levels of proviral DNA in the gut (in three both in ileum and colon, in two only in sigmoid or ileum). In the future, it will be relevant to compare HIV-2 phylogenic diversity in the blood and gut. Moreover, it will be relevant to evaluate the contribution of our finding of local expansion of IFN $\gamma$-producing CD8 T-cells to the gut containment of HIV-2 replication. Notably, we found no evidence of mucosal upregulation of type I Interferon, which is in agreement with our previous data generated using blood samples from a different cohort of HIV-2 infected individuals. ${ }^{26}$

Massive mucosal CD4 T-cell depletion is known to occur very early during acute HIV-1 infection. ${ }^{9}$ In this respect, there are no reports of HIV-2 acute infections, raising the possibility that the kinetics of viral dissemination may differ for the two viruses, and that HIV-2 plasma viral load may never reach the levels observed in HIV-1. ${ }^{21,37}$ Therefore, it is plausible that a slower rate of viral dissemination may facilitate the adequate mounting of mucosal responses enabling control of local viral 
a lleum
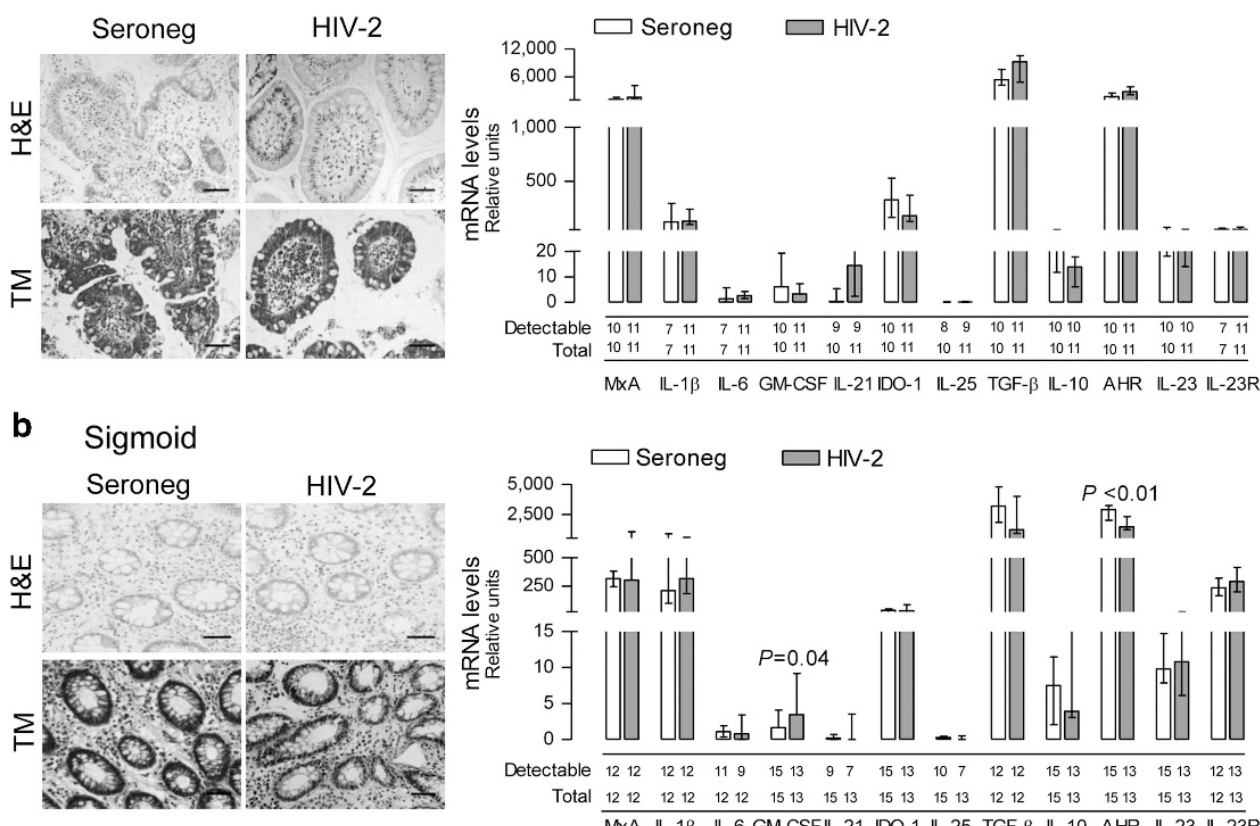

c
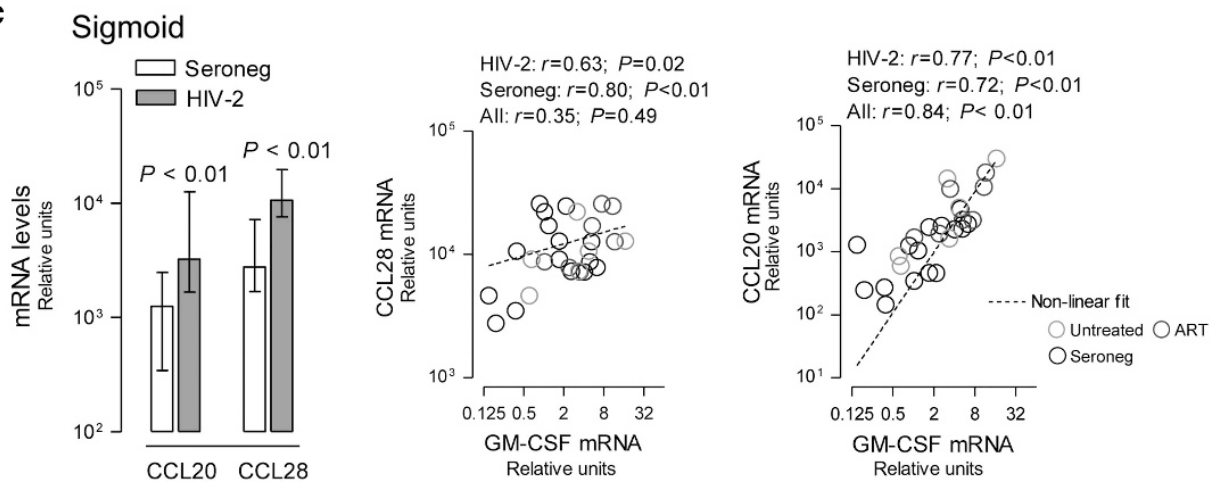

Figure 7 Preservation of the gut mucosal environment in HIV-2 infected individuals. (a-b) Representative H\&E and Masson's trichrome staining in sections of an ileum (a) and a sigmoid (b) biopsy from HIV-2-infected and seronegative individuals. (bars $=50 \mu \mathrm{m}$ ); graphs show mRNA levels of molecules involved in mucosal homeostasis in total RNA extracted from ileum (a) and sigmoid (b) biopsies from both cohorts; the total number of samples tested and those with levels above the detection threshold of the respective gene are shown below the graphs. (c) CCL20 and CCL28 mRNA levels in total RNA extracted from sigmoid biopsies of HIV-2-infected and seronegative individuals (left hand graph); and association between gene expression levels of CCL28 and GM-CSF (middle graph) and CCL20 and GM-CSF (right hand graph) in sigmoid biopsies from both cohorts. mRNA levels are expressed in relative units and normalized to the mean CT levels of GAPDH and r18S. Bars represent median values and interquartile range. Two-group comparisons were made using the Mann-Whitney $U$-test; Spearman correlation was used to calculate $r$ coefficient and statistical significance; $P$-values $<0.05$ are shown. H\&E, hematoxylin and eosin.

replication. Moreover, tissue repair mechanisms are likely to change with age and to be impaired in infancy. This might explain our previous finding of mucosal CD4 T-cell depletion in an adolescent with HIV-2 infection acquired perinatally, ${ }^{38}$ when microbial colonization of the gut is ongoing and the mechanisms underlying gut homeostasis and repair are still immature. $^{39}$

Tight junction conservation during HIV-2 infection is likely to be a key factor. In HIV-1 and SIV models, loss of tight junction integrity, via direct or indirect viral effects, is linked to overall mucosal disruption, ${ }^{31}$ increased levels of microbial translocation, and consequent systemic immune activation. ${ }^{8}$ The detailed mechanisms by which HIV-1 proteins are able to disrupt tight junctions remain unclear. ${ }^{40}$ We showed here that
HIV-2 is limited in its ability to directly impact tight junctions, providing a new model to investigate mechanisms of HIV disruption of the epithelial barrier. This has the potential to identify both new therapeutic and preventive targets, the latter to be combined with microbicides to protect the genital tract. In fact, it is plausible to speculate that the inability of HIV-2 to disrupt the epithelial barrier may, in addition to the low viremia, contribute to its low levels of sexual transmission. ${ }^{37}$

Tight junction loss in HIV-1 infection is also a consequence of high local production of pro-inflammatory cytokines, such as IL-1 and IL- $6 .{ }^{41}$ We did not find an increase in their expression levels in HIV-2 infection. It is likely that the absence of disruption of epithelial lining limits the amount of microbial products able to reach the lamina propria and, thus, prevents 
the local inflammatory responses that promote the vicious cycle that perpetuates tight junction loss in HIV-1 and SIV infections. On the other hand, it may allow the mounting of local responses mediated by IL-22 that further promote epithelial integrity.

Epithelial repair during inflammatory processes relies on multiple pathways. ${ }^{2}$ In this regard, the preservation of ILC3 in HIV-2 infection is expected to play a fundamental role, not only due to their production of IL-22 but also because they control pro-inflammatory responses by CD4 T-cells. ${ }^{42}$ Depletion of ILC3 during acute HIV-1 infection occurs both in the gut and peripheral blood, with the degree of depletion directly correlating with loss of barrier function. ${ }^{15}$ Additionally, we have previously reported preserved mucosal homeostasis in treated HIV-1 individuals with persistent $\mathrm{CD} 4 \mathrm{~T}$-cell depletion and linked this finding to a preserved ILC3 compartment. ${ }^{17}$ Importantly, in other disease settings such as chronic inflammatory bowel disease, ILC3 depletion was also found to contribute to epithelial disruption. ${ }^{43}$ Unexpectedly, HIV-2-infected individuals featured reduced transcriptional levels of $A H R$, a key regulator of IL-22 production. The possible mechanisms underlying this finding, such as microbiome imbalances, deserve further exploration. Of note, although type I interferon has been shown to negatively regulate AHR expression, ${ }^{44}$ this does not appear to be the case here, given the lack of increased in $M x A$ expression, an interferon inducible gene.

Of note, both $\mathrm{CD} 4 \mathrm{~T}$-cell numbers and quality were preserved in the gut mucosa of HIV-2-infected individuals. CD4 T-cell depletion in HIV-1 infection has been attributed to multiple processes, including direct viral-induced cytopathicity and indirect mechanisms, such as alterations in T-cell traffic. ${ }^{45}$ In fact, the levels of mucosal homing molecules in circulating CD4 T-cells, such as $\beta 7$ integrin, directly correlates with gut CD4 T-cell frequency both in humans and non-human primates. ${ }^{46}$ Our finding of increased levels of the chemokines CCL20 and CCL28, responsible for Th17 and Th22 recruitment, respectively, further support that cell recruitment plays an important role in mucosal T-cell homeostasis. Notably, we showed that this response directly correlated with increased mucosal production of GM-CSF. This cytokine is produced by several cell types, including ILC3 and is involved in maintaining a tolerogenic environment. ${ }^{47}$ Interestingly, in recent murine studies, GM-CSF was shown to be relevant in an inflammatory context by increasing T-cell homing to the gut. ${ }^{47,48}$ This supports our proposed model in which GM-CSF orchestrates an adequate mucosal response able to counteract chronic viral replication that helps maintain gut homeostasis.

Overall, we revealed for the first time that persistent viral replication occurs in the gut of chronic HIV-2-infected individuals and is associated with a local immune response able to preserve CD4 T-cells and epithelial integrity, likely contributing to the more benign course of HIV-2 disease. Importantly, our data add significantly to the understanding of mucosal CD4 T-cell preservation in the presence of chronic inflammatory stimuli, with general implications for designing new therapies aimed at restoring gut mucosal homeostasis.

\section{METHODS}

Studied cohorts. HIV-2-infected individuals with clinical indication to perform colonoscopy (Table 1) were studied and compared with a previously described control cohort. ${ }^{17}$ All individuals gave written informed consent for blood and gut biopsy collection. The study was conducted with approval of the Ethical Boards of the Faculty of Medicine of University of Lisbon, and Centro Hospitalar Lisboa Norte, Hospital São Bernardo, and Hospital Fernando da Fonseca, Portugal.

Cell isolation. Biopsies were collected from macroscopically normal mucosa, and immediately processed, as described. ${ }^{17}$ Briefly, 7-10 sigmoid biopsies/individual were digested, mechanically macerated, lymphocytes separated by Percoll gradient (Sigma-Aldrich, GE Healthcare, Saint Louis, MO, USA), and rested for $16 \mathrm{~h}$ at $37^{\circ} \mathrm{C}$ in complete medium prior to analysis. Blood was collected in parallel and PBMC isolated by Ficoll-Hypaque (Sigma-Aldrich) gradient centrifugation.

Flow cytometry. Cells were surface and intracellularly stained (Foxp3 Staining Buffer Set, EBioscience, San Diego, CA, USA), as described, ${ }^{49}$ and acquired on a LSRFortessa flow cytometer (BD Biosciences, San Jose, CA, USA). Whole-blood staining was used to assess circulating lymphocyte populations and their expression of activation markers, as described. ${ }^{26}$ Cytokine production was quantified following 4 -h stimulation with phorbol myristate acetate, plus ionomycin, in the presence of Brefeldin A (Sigma-Aldrich), using unstimulated cells to define cytokine cut-offs. ${ }^{17}$ Analysis of cytokine production was performed within ILC3 defined using ckit, NKp44, CD45, and Lin (CD3/CD14/CD16/CD19/CD123) or CD3, as well as within T-cell subsets defined using CD3, CD4, CD8, and FOXP3. The following antibodies against human (clone in brackets) were used: NKp44 (243415), CCR7 (150503) from R\&D (Minnneapolis, MN, USA); CD3 (UCHT1), CD4 (RPA-T4), CD11c (B-Ly6), CD25 (M-A251), CD45 (HI30), CD45RO (UCHL1), CD56 (My31) from BD Bioscience; CD123 (6H6), IFN $\gamma$ (4S.B3) from Biolegend (San Diego, CA, USA); CD3 (OKT3), CD4 (RPA-T4), CD8 (RPA-T8), CD14 (61D3), CD16 (eBioCB16), CD19 (HIB19), Cd38 (HB7), CD45 (2D1), CD45RO (UCHL1), Cd117 (104D2), FOXP3 (L243), IL-17 (eBIO54DEC17), IL22 (22URTI) from EBioscience. Eight-color flow cytometry analysis was performed with FlowJo (Tree Star, Ashland, OR, USA), after exclusion of dead cells (Live/Dead-LD, Thermo Fisher Scientific, Carlsbad, CA, USA) and doublets, within gated populations containing $\geqslant 150$ events, with expression of molecules normalized to unstained and/or unstimulated samples.

Immunohistochemistry staining. One sigmoid and one ileum biopsy/ individual were embedded in paraffin after preservation in $4 \%$ formaldehyde. About $3 \mu \mathrm{m}$ sections were stained as previously described, ${ }^{17}$ and revealed with Dako EnVision Detection Systems Peroxidase/DAB, Rabbit/Mouse kit (Agilent Technologies, Santa Clara, CA, USA), using the following primary antibodies (clone in brackets) against CD4 (4B12), CD8 (1A5), and FOXP3 (236/E7) from Leica Biosystems (Wetzlar, Germany); IL-17 polyclonal from Santa Cruz Biotechnology (Dallas, TX, USA); and p27 (ARP 396/397) from AIDS Research and Reference Reagent Program, Division of AIDS, National Institute of Allergy and Infectious Diseases, from Dr P Szawlowski, followed by HRP-Labeled Polymer from DAKO/Agilent Technologies. Cells with lymphocyte morphology were counted in 10 images ( $\times 400)$ acquired with a Leica DM2500 brightfield microscope, and lamina propria area assessed using ImageJ 1.47i (https://imagej.nih.gov/ij/index.html), in blinded samples by two researchers. $\mathrm{H} \& \mathrm{E}$, Masson's trichrome, and periodic acid Shiff-Alcien Blue staining were performed concurrently, and blindly evaluated by a trained pathologist. Samples were scored according to inflammatory alterations, type of infiltrate, presence or absence of fibrosis, and number of mitotic figures at the crypt base using an arbitrary scale ranging from 0 to 3 . 
Immunofluorescence staining. One sigmoid and one ileum biopsy/ individual were frozen in optimal cutting temperature compound (VWR, Radnor, PA, USA) and stained as described. ${ }^{17}$ About $3 \mu \mathrm{m}$ tissue sections were sequentially stained with antibodies after protein blocking, and 4',6-diamidino-2-phenylindole (DAPI) was used for nuclear counterstaining. Primary antibodies used were rabbit antiROR $\gamma \mathrm{t}$ (Abcam, Cambridge, UK), goat anti-IL22 (Santa Cruz Biotechnology), rat anti-CD3 (clone CD3-12 from Acris, Herford, Germany), and mouse anti-ZO-1(clone 1/ZO-1 from BD Biosciences). The respective secondary antibodies were from Molecular Probes, Thermo Fisher Scientific (Waltham, MA). Images were acquired with Zeiss LSM710 confocal point-scanning microscope (Carl Zeiss, Oberkochen, Germany) using a EC Plan-Neofluar $\times 40 / 1.30$ objective. Surface area was measured with ImageJ $1.47 \mathrm{i}$, and positive cells counted using in-house software, written in MATLAB (Mathworks, Natick, MA, USA), allowing automatic generation of individual report images for cell-counting verification. ${ }^{17}$ Regarding ZO-1 expression, the total amount was measured as a percentage of the epithelium area by Fiji, using thresholds setting for two channels: DAPI in blue and ZO-1 in green.

RNA/DNA extraction and analysis. One sigmoid and one ileum biopsy were stored in RLT buffer (Qiagen, Valencia, CA, USA) immediately post collection. RNA and DNA were extracted using Allprep RNA/DNA mini kit (Qiagen). cDNA was synthesized from $250 \mathrm{ng}$ of RNA (SuperScript III), and used to quantify the expression levels of MxA using KiCqStart primers and Power SYBR Green master mix, as well as IL22, IL17A, IL22RA1, IL-23, IL23R, IL25, TGFB1, IL1B, IL-6, IL-10, CCL20, CCL28, GM-CSF, MUC1, MUC13, S100A9, REG3G, DEFB4A, AHR, and IDO1 after pre-amplification with TaqMan Preamp Master Mix, using Applied Biosystems TaqMan gene expression assays and Applied Biosystems 7500 Fast or ViiA7 RealTime PCR Systems (all from Thermo Fisher Scientific except MxA from Sigma-Aldrich). Results are expressed using the $\Delta \mathrm{Ct}$ method (normalized to the average cycle threshold $(\mathrm{Ct})$ values of GAPDH and r18S). Cell-associated HIV-2 DNA was quantified by a nested qPCR using the following primers and probes: $\mathrm{HIV}-2$ gag out-forward 5'-GTGGGAGATGGGCGCGAAAA-3', out-reverse $5^{\prime}$-TCTCTGC TAATCCGAATCTGTC-3' , in-forward $5^{\prime}$-CGCGAGAAACTCCG TCTTG- $3^{\prime}$, in-reverse $5^{\prime}$-GCTGCCCACACAATATGTTTTA- $3^{\prime}$, probe FAM 5'-CCGGGCCGTAACCT-3' MGB; CD3 $\gamma$ out-forward $5^{\prime}$-CCAGCTCTGAAGTAGGGAACATAT-3', out-reverse $5^{\prime}$-ACTG ACATGGAACAGGGGAAG-3', in-forward $5^{\prime}$-agggcaaaatggaggctc tta- $3^{\prime}$, in-reverse $5^{\prime}$-TCTCCTCCATGGGACACTGTT-3', probe VIC-5'-CTCTCTAGCAGAGAACAGT-3'-MGB. Standard curves were generated from serial dilutions of a plasmid containing both amplicons. ${ }^{22}$ Cell-associated HIV-2 DNA was quantified in $50 \mathrm{ng}$ of total DNA purified from PBMCs or from digested gut biopsies. The first step of the PCR was carried out in a $50 \mu \mathrm{l}$ reaction mix containing the "outer" primers, dNTPs, $\mathrm{MgCl}$, Taq DNA Polymerase (GoTaq Flexi, Promega, Madison, WI), buffer, and $5 \mu$ l of DNA or of serial dilutions of the plasmid. The amplification reaction was performed using a Bio-Rad T100 Thermal Cycler (Hercules, CA), as follows: 2 min denaturation at $95^{\circ} \mathrm{C}, 20$ cycles of $95^{\circ} \mathrm{C}$ for $30 \mathrm{~s}, 60^{\circ} \mathrm{C}$ for $30 \mathrm{~s}$, and $72{ }^{\circ} \mathrm{C}$ for $2 \mathrm{~min}, 5 \mathrm{~min}$ extension at $72^{\circ} \mathrm{C}$. The reaction mix for the second step was composed of $5 \mu \mathrm{l}$ of PCR product diluted 1:50 (including standard curves), TaqMan gene expression master mix and the "in" primers and probes. Amplification was performed using a 7500 Fast Real-Time PCR system. Plasma HIV-2 viral load was quantified by RT-PCR using a previously described assay, ${ }^{37}$ with a detection cut-off of 40 RNA copies per ml.

Colon cell line cultures with HIV. Human colon-derived crypt-like T84 epithelial cell line (ATCC catalogue CCL-248) was maintained according to previously published protocols. ${ }^{31} \mathrm{~T} 84$ cells were seeded in a 4-well-chamber slide (Lab-Tek II RS glass, Nunc, Thermo Fisher Scientific) and grown for 3-4 days until reaching near confluence, as determined by light microscopy. Cells were then cultured for an additional $24 \mathrm{~h}$ with medium alone or with 10,000 TCID50 of labadapted viruses HIV-2 $2_{\text {ROD }}$ or HIV-1 $1_{\text {III }}$ (both CXCR4-tropic); or HIV2 primary isolates HIV-2 ${ }_{60,415 \mathrm{~K}}$ (CCR5-tropic) or HIV-2 20.04 (CXCR4tropic), obtained as previously described. ${ }^{22}$ Immunofluorescence staining for ZO-1 was performed and standardized 30-slice range Z-Stack acquired on confocal LSM710. Representative images are maximum intensity projections of the Z-stacks using ImageJ 1.47i.

Statistical analysis. Results are expressed as medians and interquartile range. Two-group comparisons were made using MannWhitney $U$-test or Wilcoxon Signed Rank test with Graph Prism 5.0 (GraphPad Software, San Diego, CA, USA). Non-adjusted regression analysis or Spearman correlation was used to identify associations between several parameters. Distribution of cytokine production by CD4 + T-cells was analysed with SPICE version 5.1 (downloaded from http://exon.niaid.nih.gov) using Wilcoxon Signed Rank test and partial permutation test. ${ }^{50} P$-values below 0.05 were considered significant.

\section{ACKNOWLEDGMENTS}

We would like to thank the individuals who participated in the study, as well as the physicians for their follow-up and collection of clinical data, particularly Joana Fernandes, Manuela Doroana, Sérgio Paulo, Nuno Janeiro, and Francisco Antunes from the Department of Infectious Diseases of Hospital de Santa Maria, Lisbon; the collaboration of the Gastroenterology Departments of Hospital de Santa Maria, Lisbon, and Hospital de S. Bernardo, Setúbal, for the endoscopic studies; Vânia Silva for the technical assistance in molecular biology; and Iris Caramalho and Rui MM. Victorino for the critical discussion of the manuscript. This work was supported by the Fundação para a Ciência e Tecnologia (FCT), and the Programa Operacional Ciência e Inovação 2010 (EXPL/DTP-PIC/0854/ 2013 to RBF, and PIC/IC/82712/2007 to AES), as well a Fundação Calouste Gulbenkian (P132532/2013 to AES). SMF, ARP, HNC, and RBF received FCT scholarships.

\section{AUTHOR CONTRIBUTIONS}

Study design: S.M.F., R.B.F., H.V.F., and A.E.S.; acquisition of data: S.M.F., A.R.P., P.M., H.N.C., and R.B.F.; analysis and interpretation of data: S.M.F., A.R.P., P.M., C.F., and R.B.F.; collection of clinical data: L.C., E.V., J.P., and P.P.; drafting of the manuscript: S.M.F. and A.E.S.; study supervision: A.E.S.

\section{DISCLOSURE}

The authors declare no conflict of interest.

c) 2018 Society for Mucosal Immunology

\section{REFERENCES}

1. Shen, S. \& Wong, C.H. Bugging inflammation: role of the gut microbiota. Clin. Transl. Immunol. 5, e72 (2016).

2. Yu, L.C., Wang, J.T., Wei, S.C. \& Ni, Y.H. Host-microbial interactions and regulation of intestinal epithelial barrier function: from physiology to pathology. World J. Gastrointest. Pathophysiol. 3, 27-43 (2012).

3. Mowat, A.M. Anatomical basis of tolerance and immunity to intestinal antigens. Nat. Rev. Immunol. 3, 331-341 (2003).

4. Sonnenberg, G.F. \& Artis, D. Innate lymphoid cells in the initiation, regulation and resolution of inflammation. Nat. Med. 21, 698-708 (2015).

5. Parks, O.B., Pociask, D.A., Hodzic, Z., Kolls, J.K. \& Good, M. Interleukin22 signaling in the regulation of intestinal health and disease. Front. Cell Dev. Biol. 3, 85 (2015).

6. Tsuji, N.M. \& Kosaka, A. Oral tolerance: intestinal homeostasis and antigenspecific regulatory T cells. Trends Immunol. 29, 532-540 (2008).

7. Hernandez-Santos, N., Huppler, A.R., Peterson, A.C., Khader, S.A., McKenna, K.C. \& Gaffen, S.L. Th17 cells confer long-term adaptive immunity to oral mucosal Candida albicans infections. Mucosal Immunol. 6, 900-910 (2013).

8. Brenchley, J.M. et al. Microbial translocation is a cause of systemic immune activation in chronic HIV infection. Nat. Med. 12, 1365-1371 (2006). 
9. Mehandru, S. et al. Mechanisms of gastrointestinal CD4 + T-cell depletion during acute and early human immunodeficiency virus type 1 infection. J. Virol. 81, 599-612 (2007).

10. Hunt, P.W. et al. Gut epithelial barrier dysfunction and innate immune activation predict mortality in treated HIV infection. J. Infect. Dis. 210, 1228-1238 (2014)

11. Jiang, W. et al. Plasma levels of bacterial DNA correlate with immune activation and the magnitude of immune restoration in persons with antiretroviral-treated HIV infection. J. Infect. Dis. 199, 1177-1185 (2009).

12. Kim, C.J. et al. A role for mucosal IL-22 production and Th22 cells in HIVassociated mucosal immunopathogenesis. Mucosal Immunol. 5, 670-680 (2012).

13. Schacker, T.W. et al. Collagen deposition in HIV-1 infected lymphatic tissues and T cell homeostasis. J. Clin. Invest. 110, 1133-1139 (2002).

14. Estes, J. et al. Collagen deposition limits immune reconstitution in the gut. J. Infect. Dis. 198, 456-464 (2008).

15. Kloverpris, H.N. et al. Innate lymphoid cells are depleted irreversibly during acute HIV-1 infection in the absence of viral suppression. Immunity $\mathbf{4 4 ,}$ 391-405 (2016).

16. Reeves, R.K. et al. Gut inflammation and indoleamine deoxygenase inhibit IL-17 production and promote cytotoxic potential in NKp44 + mucosal NK cells during SIV infection. Blood 118, 3321-3330 (2011).

17. Fernandes, S.M. et al. Enteric mucosa integrity in the presence of a preserved innate interleukin 22 compartment in HIV type 1-treated individuals. J. Infect. Dis. 210, 630-640 (2014).

18. Jaffar, S. et al. Rate of decline of percentage CD4 + cells is faster in HIV-1 than in HIV-2 infection. J. Acquir. Immune Defic. Syndr. 16, 327-332 (1997).

19. Sousa, A.E., Carneiro, J., Meier-Schellersheim, M., Grossman, Z. \& Victorino, RMM. CD4 Tcell depletion is linked directly to immune activation in the pathogenesis of HIV-1 and HIV-2 but only indirectly to the viral load. J. Immunol. 169, 3400-3406 (2002).

20. Grossman, Z., Meier-Schellersheim, M., Sousa, A.E., Victorino, R.M. \& Paul, W.E. CD4 + T-cell depletion in HIV infection: are we closer to understanding the cause?. Nat. Med. 8, 319-323 (2002).

21. Soares, R.S. et al. Cell-associated viral burden provides evidence of ongoing viral replication in aviremic HIV-2-infected patients. J. Virol. 85, 2429-2438 (2011).

22. Nunes-Cabaco, H. et al. Thymic HIV-2 infection uncovers posttranscriptional control of viral replication in human thymocytes. J. Virol. 89, 2201-2208 (2015).

23. Jobe, O. et al. Proviral load and immune function in blood and lymph node during HIV-1 and HIV-2 infection. Clin. Exp. Immunol. 116, 474-478 (1999).

24. de Silva, T.I. et al. Correlates of T-cell-mediated viral control and phenotype of CD8(+) Tcells in HIV-2, a naturally contained human retroviral infection. Blood 121, 4330-4339 (2013).

25. Nowroozalizadeh, S. et al. Microbial translocation correlates with the severity of both HIV-1 and HIV-2 infections. J. Infect. Dis. 201, 1150-1154 (2010).

26. Cavaleiro, R. et al. Monocyte and myeloid dendritic cell activation occurs throughout HIV type 2 infection, an attenuated form of HIV disease. J. Infect. Dis. 207, 1730-1742 (2013).

27. Drylewicz, J. et al. Comparison of viro-immunological marker changes between HIV-1 and HIV-2-infected patients in France. AIDS 22, 457-468 (2008).

28. Epple, H.J. et al. Mucosal but not peripheral FOXP3 + regulatory Tcells are highly increased in untreated $\mathrm{HIV}$ infection and normalize after suppressive HAART. Blood 108, 3072-3078 (2006).

29. Favre, D. et al. Critical loss of the balance between Th17 and Tregulatory cell populations in pathogenic SIV infection. PLoS Pathog. 5, e1000295 (2009).
30. Cella, M., Otero, K. \& Colonna, M. Expansion of human NK-22 cells with IL-7, IL-2, and IL-1beta reveals intrinsic functional plasticity. Proc. Nat/. Acad. Sci. USA 107, 10961-10966 (2010).

31. Nazli, A. et al. Exposure to HIV-1 directly impairs mucosal epithelial barrier integrity allowing microbial translocation. PLoS Pathog. 6, e1000852 (2010).

32. Qiu, J. et al. The aryl hydrocarbon receptor regulates gut immunity through modulation of innate lymphoid cells. Immunity 36, 92-104 (2012).

33. Ahlfors, H. et al. IL-22 fate reporter reveals origin and control of IL-22 production in homeostasis and infection. J. Immunol. 193, 4602-4613 (2014).

34. Monteiro, P. et al. Memory CCR6 + CD $4+$ T cells are preferential targets for productive HIV type 1 infection regardless of their expression of integrin beta7. J. Immunol. 186, 4618-4630 (2011).

35. Page, E.E. et al. Loss of Th22 cells is associated with increased immune activation and IDO-1 activity in HIV-1 infection. J. Acquir. Immune Defic. Syndr. 67, 227-235 (2014).

36. Schramm, B., Penn, M.L., Palacios, E.H., Grant, R.M., Kirchhoff, F. \& Goldsmith, M.A. Cytopathicity of human immunodeficiency virus type 2 (HIV-2) in human lymphoid tissue is coreceptor dependent and comparable to that of HIV-1. J. Virol. 74, 9594-9600 (2000).

37. Soriano, V. et al. Human immunodeficiency virus type 2 (HIV-2) in Portugal: clinical spectrum, circulating subtypes, virus isolation, and plasma viral load. J. Med. Virol. 61, 111-116 (2000).

38. Fernandes, S.M. et al. Gut disruption in HIV-2 infection despite reduced viremia. AIDS 28, 290-292 (2014).

39. Bunders, M.J. et al. Memory CD4(+)CCR5 $(+)$ T cells are abundantly present in the gut of newborn infants to facilitate mother-to-child transmission of HIV-1. Blood 120, 4383-4390 (2012).

40. Clayton, F. et al. Gp120-induced Bob/GPR15 activation: a possible cause of human immunodeficiency virus enteropathy. Am. J. Pathol. 159, 1933-1939 (2001).

41. Schulbin, H. et al. Cytokine expression in the colonic mucosa of human immunodeficiency virus-infected individuals before and during 9 months of antiretroviral therapy. Antimicrob. Agents Chemother. 52, 3377-3384 (2008).

42. Hepworth, M.R. et al. Innate lymphoid cells regulate CD4 + T-cell responses to intestinal commensal bacteria. Nature 498, 113-117 (2013).

43. Takayama, T. et al. Imbalance of NKp44(+)NKp46(-) and NKp44()NKp46(+) natural killer cells in the intestinal mucosa of patients with Crohn's disease. Gastroenterology 139, 882-892 (2010).

44. Rothhammer, V. et al. Type I interferons and microbial metabolites of tryptophan modulate astrocyte activity and central nervous system inflammation via the aryl hydrocarbon receptor. Nat. Med. 22, 586597 (2016).

45. Mavigner, M. et al. Altered CD4 + Tcell homing to the gut impairs mucosal immune reconstitution in treated HIV-infected individuals. J. Clin. Invest. 122, 62-69 (2012).

46. Ciccone, E.J. et al. Cycling of gut mucosal CD4 + T cells decreases after prolonged anti-retroviral therapy and is associated with plasma LPS levels. Mucosal Immunol. 3, 172-181 (2010).

47. Pearson, C. et al. ILC3 GM-CSF production and mobilisation orchestrate acute intestinal inflammation. Elife 5, e10066 (2016).

48. Samarakoon, A. et al. CD45 regulates GM-CSF, retinoic acid and T-cell homing in intestinal inflammation. Mucosal Immunol 9, 1514-1527 (2016).

49. Foxall, R.B. et al. Memory and naive-like regulatory CD4+ Tcells expand during HIV-2 infection in direct association with CD4 + T-cell depletion irrespectively of viremia. AIDS 25, 1961-1970 (2011).

50. Roederer, M., Nozzi, J.L. \& Nason, M.C. SPICE: exploration and analysis of post-cytometric complex multivariate datasets. Cytometry A 79, 167-174 (2011). 